\title{
Linear buckling of quadrangular and kagome gridshells: a comparative assessment
}

\author{
Romain Mesnil $^{\mathrm{a}, \mathrm{b}, *}$, Cyril Douthe ${ }^{\mathrm{a}}$, Olivier Baverel ${ }^{\mathrm{a}}$, Bruno Léger $^{\mathrm{b}}$ \\ ${ }^{a}$ Laboratoire Navier, UMR 8205, École des Ponts, IFSTTAR, CNRS, UPE, Champs-sur-Marne, France \\ ${ }^{b}$ Bouygues Construction SA, Guyancourt, France
}

\begin{abstract}
The design of gridshells is subject to strong mechanical and fabrication constraints, which remain largely unexplored for non-regular patterns. The aim of this article is to compare the structural performance of two kind of gridshells. The first one is the kagome gridshell and it is derived from a non-regular pattern constituted of triangles and hexagons. The second one results from a regular pattern of quadrangles unbraced by diagonal elements. A method is proposed to cover kagome gridshells with planar facets, which reduces considerably the cost of fabrication of the cladding.

The sensitivity of kagome gridshells to geometrical imperfections is discussed. The linearised buckling load of kagome gridshells is then compared to the one of quadrilateral gridshells. The most relevant design variables are considered in the parametric study. Two building typologies are studied for symmetrical and non-symmetrical load cases: dome and barrel vault. It reveals that the kagome gridshell outperforms quadrilateral gridshell for a very similar construction cost.
\end{abstract}

Keywords: grid shell, conceptual design, fabrication-aware design, kagome grid pattern, quadrangular grid pattern, imperfection, buckling analysis, performance assessment

\section{Introduction}

Grid-shells are structures made of beam elements that act as continuous shells structures. The choice of a grid pattern influences both fabrication and structural behaviour. For example, triangulated structures are known to be stiffer than quadrangular meshes. Quadrangular grishells rely on the bending stiffness of connections, whereas triangulated gridshells benefit from a shell-like behaviour without the need for rigid connections. The better structural performance of triangular gridshells is however at the cost of an increased node complexity due to higher node valence. In quadrangular meshes, panels are however not necessarily planar, and only specific curve networks on surfaces or shape-generation strategies guarantee meshing with planar quadrilaterals $[1,2,3,4]$. There is thus a necessary trade-off between design freedom and fabrication constraints.

This article focuses on a lesser known family of pattern, called kagome grid pattern, composed from triangles and hexagons and represented in Figure 1. The kagome pattern can be found in Japanese basketry, where the members are woven. We focus here on applications to structural engineering and consider non-woven pattern, where all the neutral axes of the beams are concomitant, and the beams are rigorously straight. Like quadrilateral grids, kagome grids present a node valence of four, which indicates a reasonable cost of fabrication. Among other usage, kagome

\footnotetext{
*Corresponding author

E-mail: romain.mesnil@enpc.fr
}

grids have been used in the architecture of Shigeru Ban and for ornamentation purpose. Their structural possibilities remain however largely unexplored, and little is known on the planarity of the facets, a key element to the economy of the envelope.

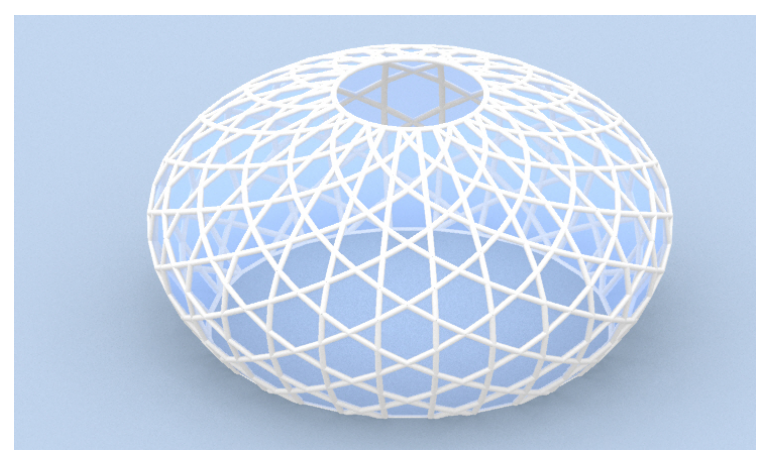

Figure 1: A kagome grid pattern covered with planar facets generated with the method described in this paper.

Kagome grid pattern and quadrilateral grid pattern have the same node valence, and their structural behaviour can be compared qualitatively. Rigid connections are necessary to guarantee in-plane shear stiffness of these patterns. However, their relatively low node valence assures the existence of a large families of torsion-free beam offsets compatible with the use of deep beams [5]. Kagome and quadrilateral grid patterns can thus be built with very similar technological solutions. Their relative structural performances is however not quantified and will be studied in this paper, whose main contributions are: 
- a strategy for the covering of kagome meshes with planar facets, demonstrating that they could be a viable alternative to triangular or quadrilateral meshes;

- a parametric study comparing the linear buckling load of kagome gridshells with quadrangular gridshells for shapes covered with planar facets;

- design guidelines for kagome gridshells.

The article is organised as follows: the first section presents the motivations for this work as well as relevant literature in the field of mechanics of gridshells. The second section introduces the methodology chosen to assess the structural behaviour of kagome gridshells. The third section gathers the results of the conducted parametric study. A brief discussion and conclusion sum up the contributions of the present work.

\subsection{Previous work on the mechanics of single-layered lat- tice shells}

The structural behaviour of gridshells is usually governed by non-linear effects, most noticeably buckling [6]. Four buckling configurations can be observed in gridshells:

- Global buckling in the manner of a shell;

- Member buckling;

- Snap-through of one node;

- In-plane rotation of one node.

Some design recommendations, often emphasizing simple shapes, like spherical cupolas have been published. Gioncu published a state of the art on the buckling of reticulated structures in 1995 [7]. A report produced by the Working Group of the International Association for Shells and Spatial Structures (IASS) in 2005 completes this review with analytical and numerical results, demonstrating the important advances made in that field [8]. A novel issue is to be published in 2016. A design guide for the stability of reticulated shells with a thorough literature review is proposed in [9], showing a great mastery of this topic.

These guidelines identify two approaches to evaluate the structural behaviour of a grid structure: homogenisation methods and numerical experiments. This article establishes a parametric numerical study, and uses previous work on homogenisation of grid structures to comment the numerical results.

\subsubsection{Homogenisation and equivalent shell thickness}

Homogenisation techniques aim to formulate an equivalent continuous behaviour of a heterogeneous structure with a cell repeated periodically. These methods use the superposition principle and usually work well for structures with a linear behaviour [10]. They have been successfully used for planar grids [11], but a rigorous extension to gridshells is difficult because of the loss of periodicity, due to the variations of curvature. A discussion on this topic is proposed by Gioncu and Balut [12].

The advantage of equivalent thickness model is that they provide structural engineers with simpler formulas and can be of practical interest for conceptual structural design. Some attempts to provide equivalent shell thickness have been used in previous studies [13, 14, 15, 16]. However, these models do not allow for the modelling of localised buckling and the study of the influence of imperfections for shell structures remains tedious for non-trivial shapes. Nowadays, the ever-growing computational power makes the use of finite element modelling and non-linear analysis ubiquitous in practice, and numerical simulations are often preferred to homogenisation formulæ.

\subsubsection{Numerical experiments}

Numerical methods are used for the practical design of gridshells, because they allow for integration of complex issues, like material nonlinearities or geometrical imperfections. Some guidelines for the analysis of reticulated domes have been proposed by Kato et al. [17, 18]: these studies introduced geometrical imperfections and semi-rigid nodes. Bulenda and Knippers [6] performed parametric studies on domes and barrels vaults and evaluated the influence of imperfections on the stability of gridshells. A more complete study using finite element analysis to evaluate local node stiffness of patented connections has been performed by Huang et al. [19]. Bruno et al. assessed the influence of nodal imperfection and of Eigenmode Imperfection Method (EIM) more recently [20]. Malek et al. [15] performed numerical investigations on the buckling of spherical cap domes and considered geometrical values, like grid spacing, or height over span ratio, as parameters. This approach lead to recommendations for the design of gridshells with triangular or quadrangular layout.

Other studies have evaluated the influence of residual stresses in elastic gridshells [16, 21]. A more complete analysis was performed on the elastic gridshell built for the Soliday's festival in Paris, considering accidental ruin of some members [22]. These studies show that high bending stresses due to the form-finding process of elastic gridshells have little influence on the buckling capacity of domes. Such procedures could be extended to steel structures, in order to assess the influence of other residual stress fields on the stability of gridshells.

\subsection{Imperfections}

There are many differences between the ideal numerical shell models and the built structures. These differences, or imperfections can be of different nature: loads, geometry, material, residual stresses in the members. Thin shells are known to be sensitive to imperfections [23]. These parameters are often set as a global geometrical imperfections. Gioncu and Balut also point out that geometrical imperfection tend to govern over material nonlinearities for large span structures [12]. 
Typically, the difference between the built geometry and the computed model is of a few centimeters at most [24]. It is therefore necessary to introduce a norm, in order to asses realistic imperfections. In the following, the norm $\|\cdot\|_{\infty}$ defined as the maximal displacement is used. Bulenda and Knippers propose a higher bound of $L / 500$ for the imperfection with the infinity norm [6]. Based on data on the precision requirements for built project [25], Malek et al. studied an imperfection of $3 \mathrm{~mm}$ [15].

The choice of the shape function is discussed in Section 2.4. The first buckling mode is recommended by design codes, and was used for example for the design of the roof of the British Museum and the Palacio de Comunicaciones $[26,25]$. However, different studies show that other imperfections shapes should be considered, as they result in a bigger reduction of the buckling capacity of gridshells. Examples of such shapes can be found in [6] with the use of dynamic eigenmodes, and a discussion on the choice of appropriate imperfections is proposed in [20]. It has to be noticed that there is no closed-form solution on the worst imperfection possible, some studies even demonstrated that higher order eigenmodes can have a more critical effect on the reduction of buckling capacity [27]. The purpose of this paper being to compare relative performance between kagome grid pattern and quadrilateral pattern, we will consider the imperfections most commonly used in current practice and limit the sensitivity analysis of section 2.4 to imperfection shapes following the first eigenmode.

\section{Methodology}

\subsection{Numerical experiment and choice of the parameters}

Two typical free-form structures are barrel-vaults and domes. Theses shapes are easily generated using translation or scale-trans surfaces surfaces, which have the advantage of generating planar quadrilateral facets. A method to convert such meshes to planar kagome meshes is described in 3.1 .

The dome is a surface of translation defined with two parabolæ.

$$
z(x, y)=\frac{4 h}{L^{2}}(y-L) \cdot y+\frac{4 H}{d^{2}}\left(x-\frac{d}{2}\right)\left(x+\frac{d}{2}\right)
$$

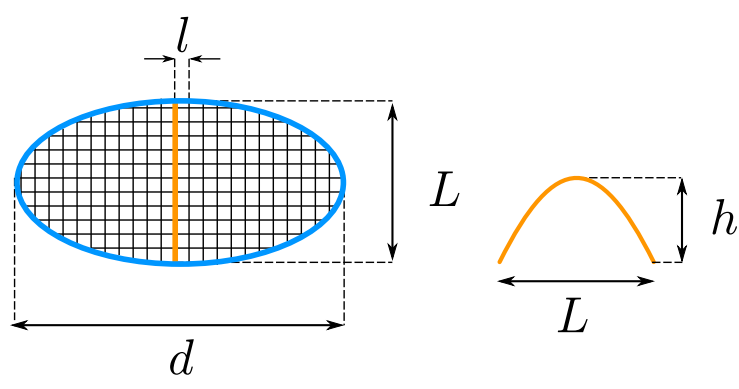

Figure 2: Geometrical parameters describing the dome.
The barrel vault is a scale-trans surface. The curves on the ground are sine curves, and the elevation is a parabola. We write $f(x)=d \sin \frac{2 \pi x}{L_{1}}$, the equation of the surface follows:

$$
z(x, y)=\frac{4 h\left(1-\frac{2 \cdot f(x)}{L}\right)}{L^{2}}(y-f(x))(y-L+f(x))
$$

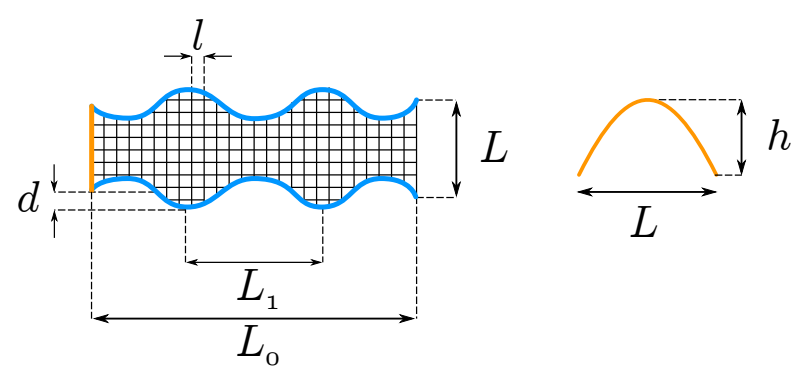

Figure 3: Geometrical parameters describing the barrel vault.

The geometrical parameters describing the two models are displayed in Figures 2 and 3. The number of geometrical parameters is quite important, we decrease their numbers by introducing non-dimensional parameters. The physical meaning of these ratios is explained and detailed below. The main span $L$ of the structures is set to 30 meters. Three ratios $\Pi_{1}, \Pi_{2}$ and $\Pi_{3}$ correspond to geometrical parameters. The two ratios $\Pi_{4}$ and $\Pi_{5}$ are the performance metrics studied in this article.

\section{Aspect ratio}

The geometry has a main span $L$ and another characteristic length $d$. The first ratio is called aspect ratio and is defined by equation (3). For the domes, the ratio $\Pi_{1}$ correspond to the ratio of curvatures, whereas for the barell vault, higher values of $\Pi_{1}$ correspond to higher gaussian curvature (the case $\Pi_{1}=0$ is a cylinder).

$$
\Pi_{1}=\frac{d}{L}
$$

Notice that different aspect ratios could be constructed from the barrel vault. For the simplicity of the demonstration, it was decided to set the ratio $L_{0} / L$ to 4 and the ratio $L_{1} / L_{0}$ to 2.5 . These values are similar to the configuration of the gridshell roof covering the museum of Downland [28].

\section{Rise-over-span ratio}

The name is self-explanatory: the second nondimensional parameter is the ratio of the characteristic height $h$ with respect to the main span $L$. Common formulas indicate that structural performance should increase with this number.

$$
\Pi_{2}=\frac{h}{L}
$$




\section{Structural density ratio}

We consider here the grids to have a mean member length of $l$. The comparison of this number to the main span, as done in equation (5) gives indications on the grid coarseness.

$$
\Pi_{3}=\frac{l}{L}
$$

\section{Buckling ratio}

The last parameter compares the buckling pressure $p_{c r}$ found by linear buckling analysis to the member bending stiffness $E I / L^{4}$. The number described by equation (6) is the value that is compared between kagome and quadrilateral meshes. The ratio $I / L^{4}$ being kept constant in this study, the buckling ratio will be a measure of the stiffness due to the form and mesh topology independently of the section properties.

$$
\Pi_{4}=\frac{p_{c r} L^{4}}{E I}
$$

Notice that only the quadratic moment of inertia $I$ is considered. A comparable non-dimensional number could be constructed with the span $L$, the axial stiffness $E A$ and the critical pressure $p_{c r}$. However, it is a well-known fact that member shortening has more impact on very shallow structures which won't be considered in our study.

\section{Structural efficiency}

We introduce finally a variable, later called structural efficiency, in order to compare the performance of kagome and quadrilateral grid pattern. The parameter is defined as:

$$
\Pi_{5}=\frac{p_{c r} \cdot A}{m \cdot g}
$$

where $A$ is the horizontal surface covered, $m$ the mass of the structure and $g$ the acceleration due to gravitational forces on Earth. The number defined by equation (7) compares the total resultant of vertical forces to the resultant of gravity forces. It must be noticed that for a same structural density, i.e. individual member length, the total length of members differs between the kagome and quadrilateral grid. For a square grid with edge length $l$, the total beam length per unit area is $\frac{2}{l}$. For a kagome grid made of regular hexagons and triangles and edge length $l$, the total beam length per unit area is $\frac{\sqrt{3}}{l}$. From this simple case, an estimation of the ratio of the masses is given by:

$$
\frac{m_{\text {Kagome }}}{m_{\text {Quad }}} \sim \frac{\sqrt{3}}{2} \simeq 86 \%
$$

In other terms, for a same structural density, the kagome grid is slightly lighter than the quadrilateral grid. This difference justifies the fact to look more closely at the structural efficiency, and not only at the buckling load.

Table 1 sums up the range of variations of each parameter. It is chosen to fit existing designs: for example the rise-over-span ratio remain in general superior to
0.1 to avoid high bending stresses or snap-through. The structural density are chosen so that the minimal member length is 1.253 , a reasonable value compared to built projects. Each set of geometrical parameters generates a geometry for a quadrilateral and a kagome grid. Two load cases are considered, as discussed in Section 2.2. The parametric study proposed in this paper consists thus of 500 linear buckling analysis and several fully nonlinear analysis for the study on imperfections sensitivity.

\subsection{Material, loads and boundary conditions}

The material used is steel, and we restrict our study to a linear elastic material law. Detailed studies with plasticity have been made previously and are reviewed in [8]. These studies are necessary to evaluate with high fidelity the post-buckling behaviour of gridshells, at the cost of high computational effort. In the first steps of the design process, engineers need to perform many analyses, often with simplified assumptions and a linearised buckling load is already a good indicator of the structural performance. It was already chosen as design criterion in [15] and [21]. The modelling hypothesis follow:

- the supports are pin joints with full translational restraint;

- the joints are assumed to be fully rigid;

- in the barrel vault, the arches are simply-supported;

- distributed loads are replaced by concentrated loads at connections.

The members are made of circular hollow section, with a wall thickness of $10 \mathrm{~mm}$ and a diameter of $200 \mathrm{~mm}$ for the dome and the barrel vault. With these geometries, there is no difference between $I_{y}, I_{z}$, and torsional buckling of members is not possible, which simplifies the parametric study. In the followings, we use beam elements with three elements per member. This subdivision allows to capture eventual localised buckling modes, which can arise in gridshells.

Two load cases are considered: a uniform projected vertical load of $1 \mathrm{kPa}$ and a non-symmetrical load of $1 \mathrm{kPa}$ applied following the normal of the surfaces with the pattern of Figure 4.

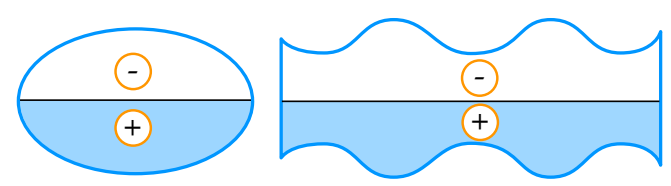

Figure 4: Areas of positive and negative pressure for the nonsymmetrical load case, top view of Figure 2.

Current literature focuses more on uniform symmetrical load cases [15]. Koiter has shown than spherical caps are subject to geometrical imperfections for such load case, but not for concentrated load. Therefore, it is meaningful 


\begin{tabular}{|c|c|c|c|}
\cline { 2 - 4 } \multicolumn{1}{c|}{} & $\Pi_{1}$ & $\Pi_{2}$ & $\Pi_{3}$ \\
\hline Barrel Vault & {$[0,0.025,0.05,0.075,0.1,0.125,0.15]$} & {$[0.1,0.2,0.3,0.4,0.5]$} & {$\left[\frac{1}{24}, \frac{1}{16}, \frac{1}{12}\right]$} \\
\hline Dome & {$[1,1.33,1.67,2]$} & {$[0.1,0.2,0.3,0.4,0.5]$} & {$\left[\frac{1}{32}, \frac{1}{24}, \frac{1}{16}\right]$} \\
\hline
\end{tabular}

Table 1: Variations of the parameters in the present study.

to consider this kind of load case in our sensitivity analysis. Furthermore, non-symmetrical load cases are known to be more critical than symmetrical ones for buckling and often govern the sizing of gridshells. The asymmetrical load case is thus also considered in order to provide guidance on situations closer to the engineering practice. The chosen asymmetrical load represents here a wind load, which usually features areas of positive and negative pressure. Wind loads computed from the Eurocode can usually be decomposed between a symmetrical and asymmetrical component. Since we already study a symmetrical load case, we focus only on the non-symmetrical component of this load.

\subsection{Buckling analysis}

This study mainly adopts linear buckling analysis of perfect gridshells. In addition, geometric non-linear analysis on structures with imperfections are carried out to preliminary evaluate the effects of imperfections. In linear buckling analysis, it is often considered that the stiffness matrix of a structure can be written as the sum of $\mathbf{K}_{E}$ the elastic stiffness (independent of the applied load $\mathbf{P}$ ) and of $\mathbf{K}_{G}$ the geometric stiffness (which decreases here with $\mathbf{P})$. The linear buckling analysis makes the assumption that the coefficients of $\mathbf{K}_{G}$ vary linearly with the amplitude of $\mathbf{P}$ and finds thus couples of buckling factor and displacement vector $(\lambda, \boldsymbol{\Phi})$ so that:

$$
\left(\mathbf{K}_{E}+\lambda \mathbf{K}_{G}\right) \cdot \boldsymbol{\Phi}=\mathbf{0}
$$

The non-linear buckling problem becomes therefore the eigenvalue problem shown in equation (9), the lowest eigenvalue $\lambda_{1}$ giving an estimate of the buckling capacity of the structures. The linearisation hypothesis is in fact a Taylor development, and it is valid if the displacements before buckling are small. In structures subject to large deformations, like gridshells, the linear buckling analysis can overestimate largely the real buckling capacity. In detailed design, fully non-linear analysis is thus required to assess the bearing capacity of gridshells, but the linear buckling analysis can be quickly estimated and can be helpful in conceptual design stage [15].

The analysis software used is Karamba, a plug-in integrated with parametric CAD tools Rhinoceros ${ }^{\mathrm{TM}}$ and Grasshopper $^{\mathrm{TM}}[29,30]$. This software enables to perform structural analysis within a 3D-modelling environment, which considerably eases the design process for structural engineers.

\subsection{Influence of imperfections}

This section focuses on the influence of imperfection on kagome gridshells. The tested geometry is a dome supported on a circular plan $\left(\Pi_{1}=1, \Pi_{2}=0.2, \Pi_{3}=1 / 32\right)$, and subject to a uniform vertical load. Figure 5 shows different plots of linear buckling load $p_{c r}$ normalised by the linear buckling load of the structure without imperfection $p_{c r, 0}$ computed with different imperfection amplitudes for the infinity norm. The kagome pattern is more sensitive to imperfections than the quadrangular pattern. For the amplitude of $L / 500$, the reduction of the linearised buckling load is approximately $10 \%$.

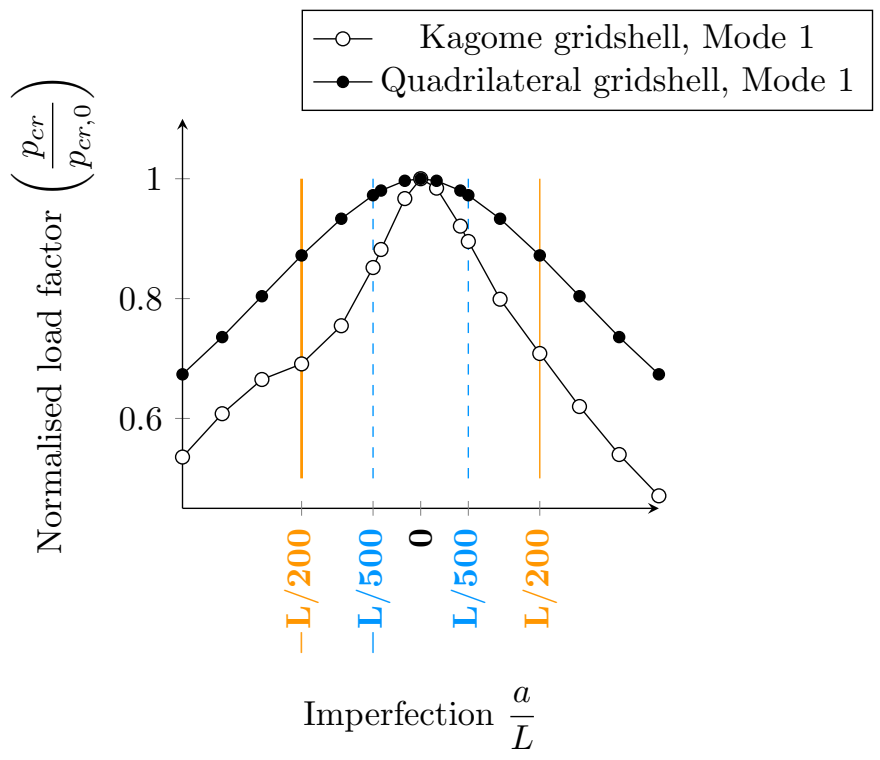

Figure 5: Influence of imperfection scale on the linear buckling load.

A second order analysis is thus performed on both perfect and imperfect geometry to evaluate more precisely the influence of imperfections. The load/displacement diagrams for the kagome and quadrilateral gridshells obtained are displayed in Figure 6 and 7 respectively. The four horizontal lines represent the linearised buckling loads. Three imperfection amplitudes are considered: the first one is a small imperfection $(L / 1500)$ and can be compared to the one used by Malek et al. [15], the second corresponds to the $(L / 500)$, as proposed by Bulenda and Knippers [6], the third one is of $(L / 200)$ as recommended in EC3.

The structures with imperfections do not reach their linearised buckling loads, contrary to perfect structures. The load/displacement graphs are less curved than the 
ones of the gridshells without imperfection. Consider for example the imperfect geometry with a norm of $15 \mathrm{~cm}$ $(L / 200)$ : the structure behaves in a fully non-linear manner and it is hard to distinguish a linear domain. This indicates that the linearised buckling load is not suited for structures with high imperfection norm, as high stresses are at stake before buckling. Considering the imperfection amplitude of $L / 500$ proposed by Bulenda and Knippers [6], we notice that the bearing capacity of the imperfect structure decreases by approximately $15 \%$. With the norm proposed by Malek et al. [15], the loss of bearing capacity with imperfections is negligible for the considered geometry.

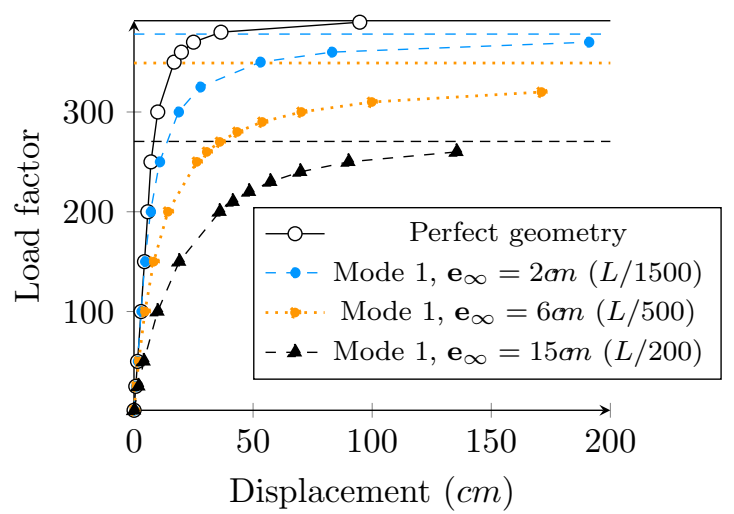

Figure 6: Load/displacement diagram for a dome covered with a kagome grid pattern.

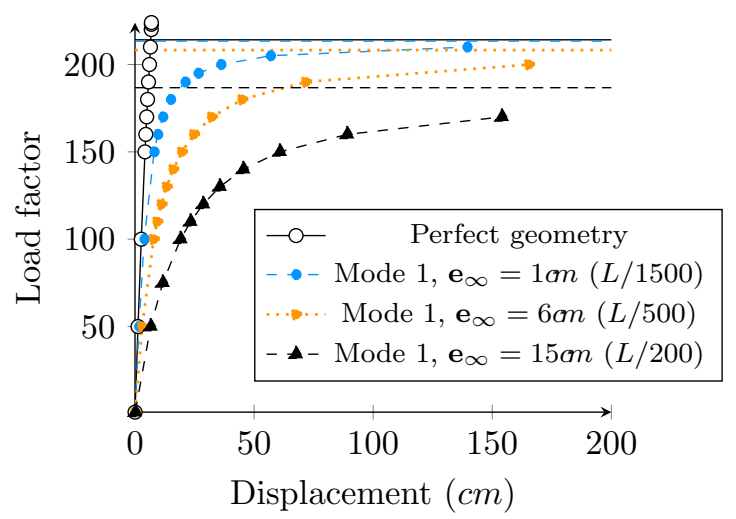

Figure 7: Load/displacement diagram for a dome covered with a quadrilateral grid pattern.

The qualitative behaviour of kagome gridshells with respect to imperfections is similar to what has been analysed in previous research on quad pattern $[6,15]$, and Figure 6 and 7 are indeed similar. In the treated example, the decrease of bearing capacity with respect to imperfection amplitude is however less important than in previous literature, for example in [6] who considered cable braced quadrilateral gridshells. It can however be compared to the decrease observed in a previous study for quadrilateral gridshells [15]. The explanation given by Malek et al. is that quadrilateral (and kagome) gridshells rely on in-plane bending stiffness of the members to withstand loads, whereas triangulated gridshells can transfer out-ofplane loads with axial forces in the members. Small imperfections can therefore introduce bending moments in triangular gridshells and change their load transfer mechanism, from axial forces to axial and bending combined.

To go further, we propose to study the load where the displacement reaches the service limit state. This displacement is found with a second order analysis. We set $\delta_{S L S}=\frac{L}{200}$ and compare the influence of imperfection for quad and kagome gridshells. Figure 8 shows the critical non-dimensional service load defined as $\frac{p_{S L S} L^{4}}{E I}$. Kagome grids remain stiffer regardless of the imperfection. This additional criterion shows also that the imperfection does not change the relative performance of kagome and quadrilateral grids for simple performance metrics used in preliminary design.

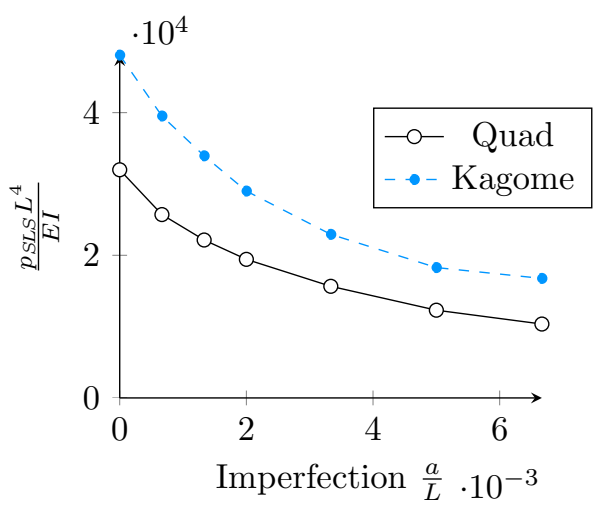

Figure 8: Non-dimensional $S L S$ load for Kagome and Quad gridshells

In summary, this section discussed the influence of imperfection on the structural performance of gridshells. The study of sensitivity to imperfection suggests that kagome and quadrilateral gridshells without cable-bracing have qualitatively similar behaviours for linear buckling analysis. Even classical approaches, like Eigenmode imperfection, illustrate the limitations of linear buckling analysis for detailed stages of design. In the following, we propose nonetheless to compare the bearing capacity of kagome and quadrilateral gridshells by studying the linearised buckling load without imperfections, because this performance indicator is commonly used in conceptual design stages. In detailed design, geometrical and material non-linear analysis will be required to assess the structural response of gridshells with full accuracy.

\section{Generation of planar kagome grid pattern}

\subsection{An algorithm for kagome pattern with planar facets}

We present here an original method that converts planar quadrilateral (PQ) meshes to planar kagome (PK) meshes. This method guarantees a control of the cost of 
the cladding, which is an important issue in free-form architectural design.

The algorithm takes a PQ-mesh as an input, like illustrated in Figure 9. Not all PQ-meshes are acceptable, but only those which can be coloured as a chequerboard. In the algorithm, the dark faces become hexagons and the white one become triangles. Starting from a quad mesh (left), one must determine intermediary points (middle) which define new vertices of the kagome grid (right).
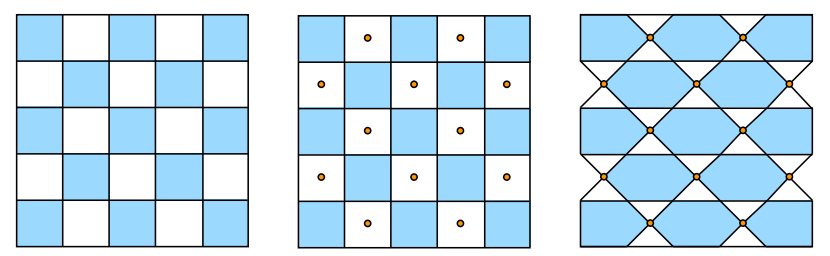

Figure 9: Conversion of a quadrilateral mesh to a kagome mesh

The choice of the intermediary point is restricted by the fact that the two adjacent hexagons have to be planar. Consider three consecutive planar quads $Q_{i-1}, Q_{i}$ and $Q_{i+1}$. The algorithm determining the new vertices can be written as follows, and is detailed in Figure 10:

1. Compute the barycentre $\mathbf{G}_{\mathbf{i}}$ of the quadrangle $Q_{i}$;

2. Compute the intersection of the planes $\left(Q_{i-1}\right)$, $\left(Q_{i+1}\right)$

- If the intersection is a plane, create the node $\mathbf{N}_{\mathbf{i}}=\mathbf{G}_{\mathbf{i}}$

- If it is a line $(\mathcal{L})$, create the node $\mathbf{N}_{\mathbf{i}}$ as the orthogonal projection of $\mathbf{G}_{\mathbf{i}}$ on $(\mathcal{L}) . \mathbf{N}_{\mathbf{i}}$ is the closest point to $\mathbf{G}_{\mathbf{i}}$ on $(\mathcal{L})$.

3. Repeat steps 1 and 2 in a chequerboard pattern.

Other points on $(\mathcal{L})$ could be chosen, but the choice proposed in this algorithm yields satisfactory and regular results, as illustrated in Figure 1.

Finally, we noticed in our formal explorations that the algorithm can encounter some difficulties if the curvature of the surface is very low. With numerical imprecisions, the binary choice of the second step of the proposed algorithm can lead to instabilities. Therefore, we introduce a number $\varepsilon$ corresponding to the fabrication tolerance for planarity. If the distance between $\mathbf{G}$ and each of the two planes is inferior to $\varepsilon$, we set the point $\mathbf{G}$ as a vertex of the kagome mesh.

\subsection{Generality of the method}

The previous algorithm gives a systematic method to generate PK-meshes. Using the work by Liu et al. [31], we can transcribe this result into notions of smooth differential geometry. They prove indeed that planar quadrilateral meshes correspond to parametrisations $(u, v)$ of surfaces satisfying a simple equation:

$$
\operatorname{det}\left(\partial_{\mathbf{u}}, \partial_{\mathbf{v}}, \partial_{\mathbf{u v}}^{\mathbf{2}}\right)=0
$$

The curves networks satisfying this equation are called conjugate-curve networks. Notice that equation (10) is satisfied by lines of curvatures as $\partial_{\mathbf{u v}}^{\mathbf{2}}=\mathbf{0}$. Therefore, any surface admits conjugate curve networks. This means that the method proposed in this article can be applied on any shape. Practically, the post-rationalisation techniques used in [32] or bottom-up techniques like [33] can be used to find conjugate curve networks on free-form shapes. Kagome meshes laid along these networks will therefore be close to PK-meshes.

\subsection{Applications to gridshells}

Several strategies for shape generation of gridshells with planar facets have been employed in the past. Among them, surfaces of revolution, scale-trans surfaces [1] or moulding surface, which generalise the notion of surface of revolution [2]. They can be combined with our conversion algorithm to generate kagome meshes with planar facets.

It must be noticed here that the kagome mesh obtained from a square grid in Figure 9 is irregular: the hexagons seem a bit stretched. Simple trigonometric considerations show that the regular kagome pattern in the plane is obtained from a rectangular grid with an aspect ratio of $\sqrt{3}$. The grids generated in this paper use the same rule, as we aim for visually regular patterns, like the ones displayed in Figure 1 and 18. Some simple geometrical properties of these grids are discussed in Appendix A.

\section{Stability of kagome gridshells}

\subsection{Buckling of barrel vaults}

Linear buckling analyses were performed on barrel vaults with different geometrical configurations under symmetrical loading, and the results are shown in Figure 12 in a non-dimensional form. In Figure $12 \mathrm{a}, \Pi_{1}=0$ and there is no corrugation, while in Figure $12 \mathrm{~b} \Pi_{1}=0.15$ and the shape is ondulating like the one shown in Figure 11. We notice that the corrugation is significantly improving the structural behaviour. The case $\Pi_{1}=0.15$ has a buckling load almost four times higher than the cylinder (case $\left.\Pi_{1}=0\right)$. The best design is shown in Figure 11 .

It appears that, in general, kagome grids have a higher buckling load. In order to quantify this assertion, we introduce the number $r$, later called ratio of efficiency, defined by equation (11). The same parameters values are chosen identical for both grids. A ratio superior to 1 indicates that the kagome gridshell is more efficient than the quadrilateral gridshell.

$$
r\left(\Pi_{1}, \Pi_{2}, \Pi_{3}\right)=\frac{\Pi_{5, \text { Kagome }}\left(\Pi_{1}, \Pi_{2}, \Pi_{3}\right)}{\Pi_{5, \text { Quad }}\left(\Pi_{1}, \Pi_{2}, \Pi_{3}\right)}
$$

In the following, $\Pi_{1}, \Pi_{2}$ and $\Pi_{3}$ have been varied and results are shown in Figure 13. We have chosen to represent the buckling load in terms of $\Pi_{1}$ and to compare the best design of both structures defined by equation (11). 


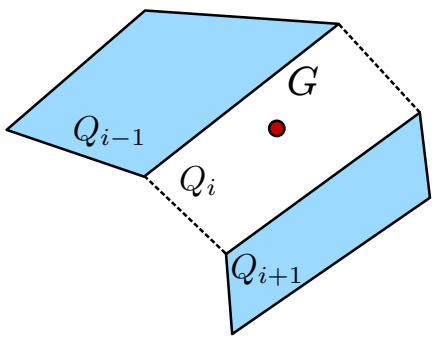

(a) Step 1

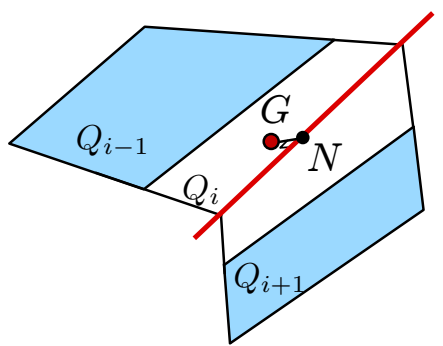

(b) Step 2

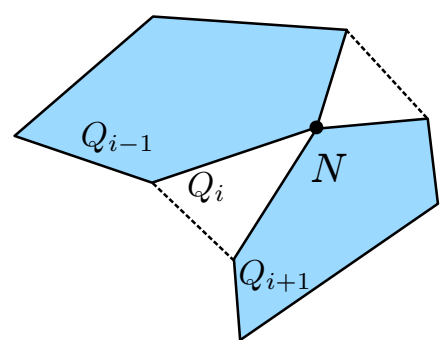

(c) Creation of the new edges

Figure 10: Details of the conversion to a Planar Kagome mesh.

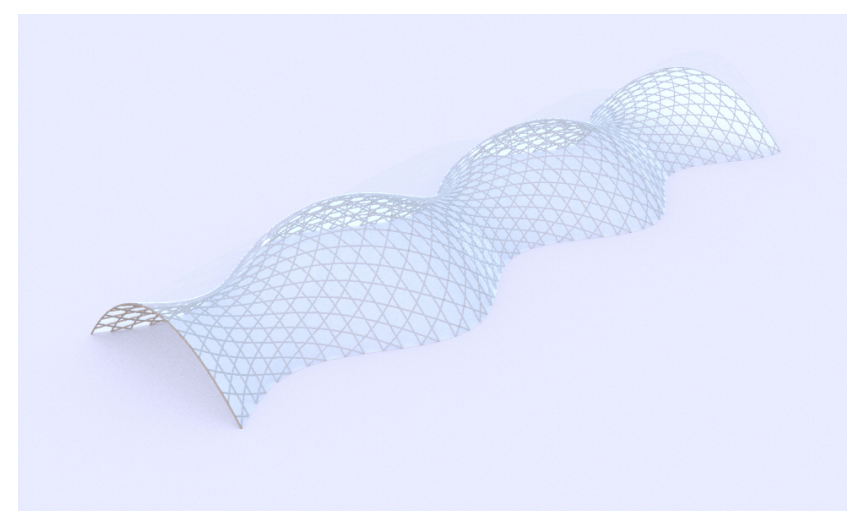

Figure 11: Optimal barrel vault: $\Pi_{1}=0.15, \Pi_{2}=0.3, \Pi_{3}=\frac{1}{24}$.

This ratio remains above 1.5 , with a peak at 2.6. The most efficient designs correspond to moderate rise-overspan ratio $\left(\Pi_{2}=0.3\right)$ and a dense grid.

$$
\left\{\begin{array}{rr}
r_{\text {min }}\left(\Pi_{1}\right)= & \min _{\Pi_{2}, \Pi_{3}} r\left(\Pi_{1}, \Pi_{2}, \Pi_{3}\right) \\
r_{\max }\left(\Pi_{1}\right)= & \max _{\Pi_{2}, \Pi_{3}} r\left(\Pi_{1}, \Pi_{2}, \Pi_{3}\right) \\
r_{\text {best }}\left(\Pi_{1}\right)= & \frac{\max _{\Pi_{2}, \Pi_{3}} \Pi_{5, \text { Kagome }}\left(\Pi_{1}, \Pi_{2}, \Pi_{3}\right)}{\max _{\Pi_{2}, \Pi_{3}} \Pi_{5, \text { Quad }}\left(\Pi_{1}, \Pi_{2}, \Pi_{3}\right)}
\end{array}\right.
$$

\subsection{Barrel vaults under non-symmetrical loads}

Non linear analysis with non-symmetrical loads were then considered with the distribution shown in Figure 4. The behaviour of the structure is then dominated by bending and becomes very different for both structures. Consider Figure 14: the quadrilateral grid has a higher buckling load, but the buckling occurs for a high level of displacements, superior to $20 \%$ of the span. Of course, the ruin of members will occurs before the structure buckles and the results on linear buckling analysis would be subject to caution for the quadrilateral grid in this case.

This is a general situation: under non-symmetrical loads, quad gridshells are considerably softer than kagome gridshells. Considering the large displacements of the quadrilateral gridshells under non-symmetrical loads, it did not seem relevant to display the results on linear buckling analysis for this load case. For the studied example, the kagome grid is indeed 5 times stiffer. Using the same SLS criterion than previously $\left(\frac{L}{200}\right)$, the kagome grid clearly outperforms the quadrilateral grid.

\subsection{Buckling of domes}

The same parametric study is then reproduced for the dome geometry. Figure 15 shows the non-dimensional buckling loads computed for the symmetrical load case. Kagome and quad grids have a similar behaviour: the buckling load is a decreasing function of $\Pi_{3}$. For slender domes (smaller values of $\Pi_{2}$ ), increasing the height also increases the buckling load, but a maximum is reached when $\Pi_{2}$ is approximately 0.3 (this optimal value of $\Pi_{2}$ depends on the cross-section used). The buckling becomes then more localised, and a change of the shape has lower impact on the buckling.

Figure 16 shows then $r_{\min }, r_{\max }$ and $r_{\text {best }}$ for different values of $\Pi_{1}$. It is noticed that the kagome gridshell is more efficient than the quadrilateral gridshell in the sense of equation 11 and this for all the configurations considered in the present study. The gain in structural efficiency is very important, especially for domes with a moderate aspect ratio, when the shell is the most efficient. It can be concluded that kagome gridshells are more efficient than quadrilateral gridshells when considering linear buckling analysis. The most interesting geometrical configurations (moderate rise-over-span, and small aspect-ratio) are also the ones where the relative performances of the two meshes typologies differ the most. The efficiency can be doubled in those cases.

Finally, Figure 17 compares the performance of kagome and quadrilateral gridshells for nonsymmetrical load cases with linear buckling analysis. The kagome gridshell remains more efficient in all cases, with a minimum gain in structural efficiency of $23 \%$. The tendency is inverted compared to the symmetrical load cases: kagome grids are more efficient when the ratio $\Pi_{2}$ is higher. 


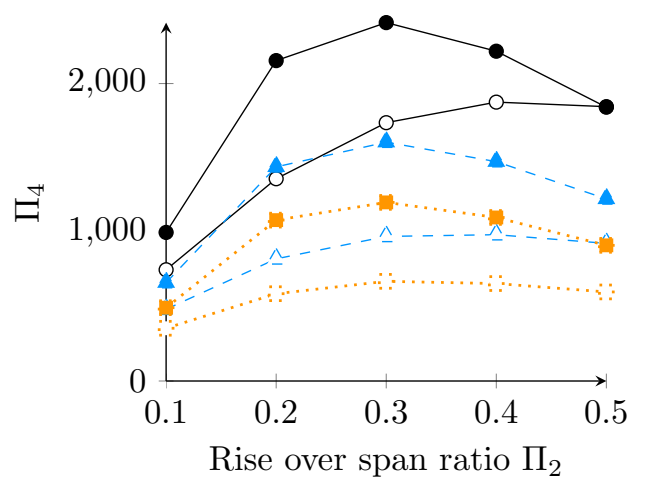

(a) Cylinder: $\Pi_{1}=\frac{d}{L}=0$

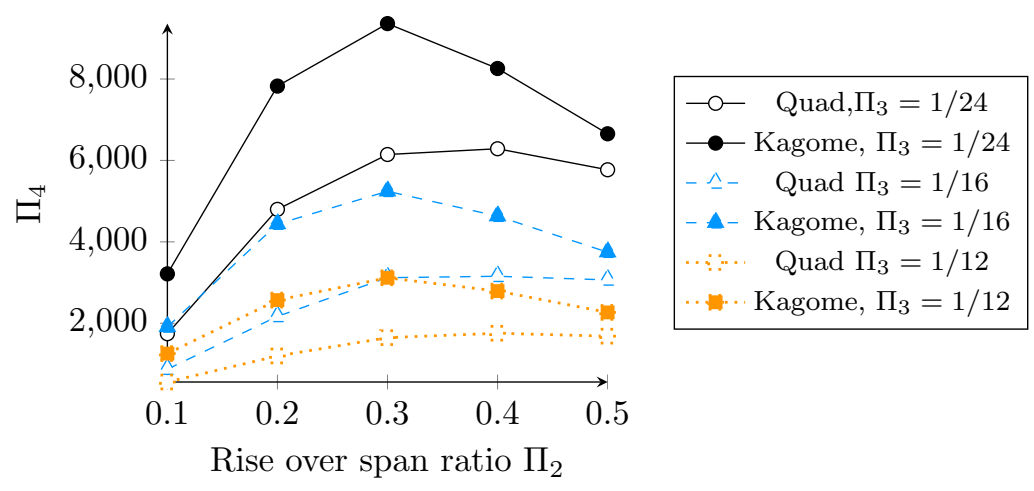

(b) Corrugated barrel vault: $\Pi_{1}=\frac{d}{L}=0.15$

Figure 12: Comparison of the buckling capacity of kagome and quadrilateral grids for the barrel vault geometry.

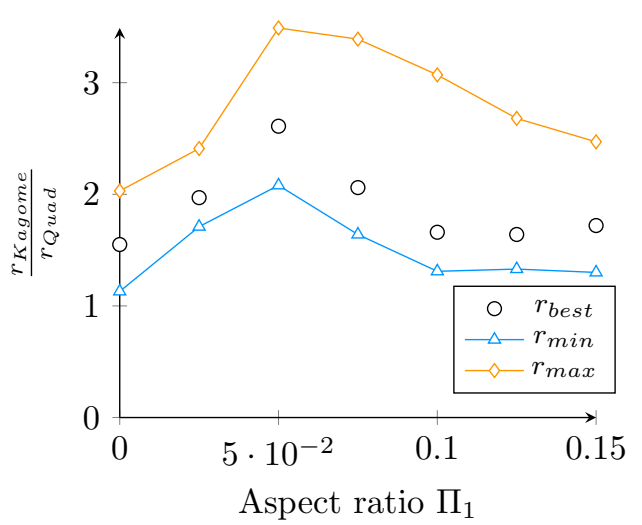

Figure 13: Comparison of the best designs for different values of $\Pi_{1}$.

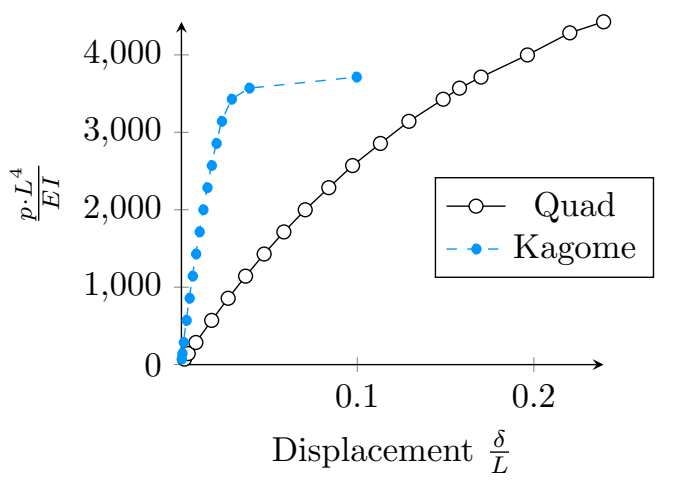

Figure 14: Load-displacement for a non-symmetrical load $\left(\Pi_{1}=0.075, \Pi_{2}=0.3, \Pi_{3}=\frac{1}{24}\right)$

\section{Discussion}

\subsection{Shape of buckled domes}

A more detailed look at the parametric study shows that the nature of buckling modes differs between kagome and quadrilateral gridshells. Figure 18 illustrates the first buckling modes for two domes with the same member length, both for quadrilateral and kagome meshes. On these images, darker colours indicate larger displacements. Each dark spot corresponds to an 'anti-node' on the buckled shape. Counting these spots, it can be noticed that the number of anti-nodes is higher in the kagome grid, and that the wavelength is shorter. This difference has been observed for coarse and fine grids.

This difference illustrates the fact that kagome gridshells have a higher in-plane shear stiffness than quadrangular meshes. Their higher buckling capacity can be explained by the fact that they activate buckling modes with shorter wavelength. This remark also holds for barrel vaults. Figure 19 shows the same kind of phenomenon for the buckling modes of the most efficient designs of barrel vaults of our study. There is the same number of antinodes in Figure 19a and 19b, but the anti-nodes are more concentrated in the kagome grid.

\subsection{Influence of mesh refinement}

The results of the previous section indicate that refining of meshes (diminishing $\Pi_{3}$ ) improves the critical buckling load of gridshells. We show a more detailed analysis of this statement by studying a dome with $\Pi_{1}=1.33$. The convergence of the structural efficiency with respect to the number of cells is interpreted with homogenisation principles and analytical formulæ from [11] and [34] and detailed in Appendix B

Consider a unit cell with characteristic length $l$ (defined in Figure 2 and 3). If one builds an equivalent shell, it is meaningful to consider that the bending and axial stiffness $\mathcal{D}$ and $\mathcal{A}$ depend linearly on $1 / l$ (doubling the number of beams would double the bending stiffness). This is found 


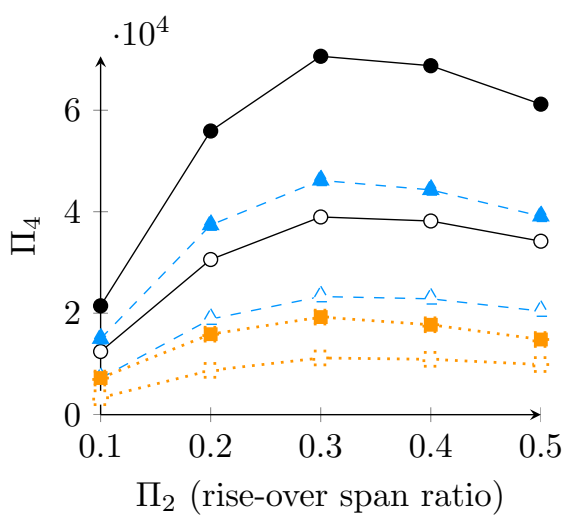

(a) $\Pi_{1}=\frac{d}{L}=1$

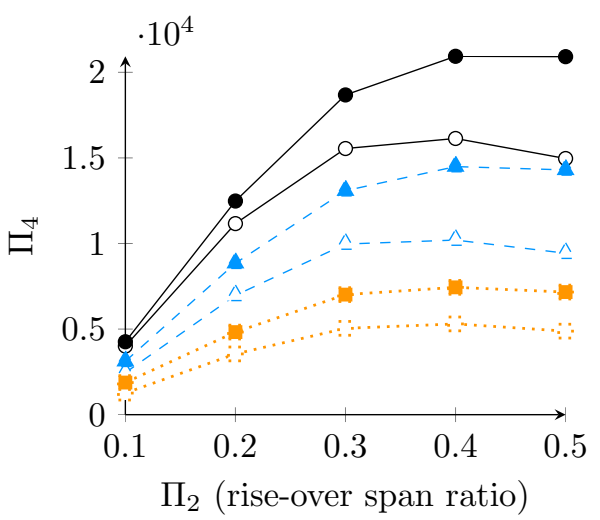

(b) $\Pi_{1}=\frac{d}{L}=2$

Figure 15: Comparison of the buckling capacity of kagome and quadrangular gridshells for the dome geometry.

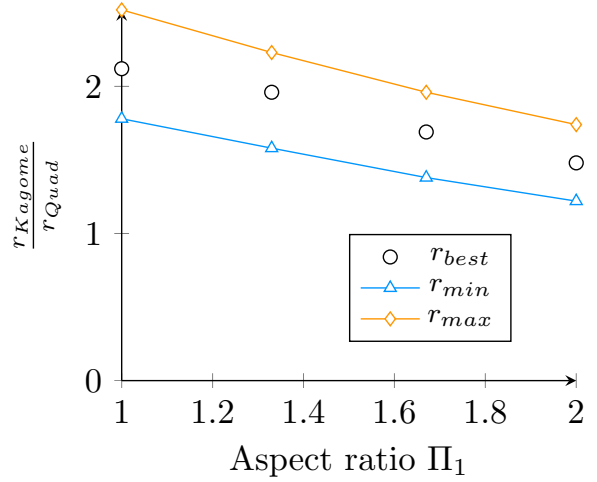

Figure 16: Comparison of the structural performance of domes under symmetrical load.

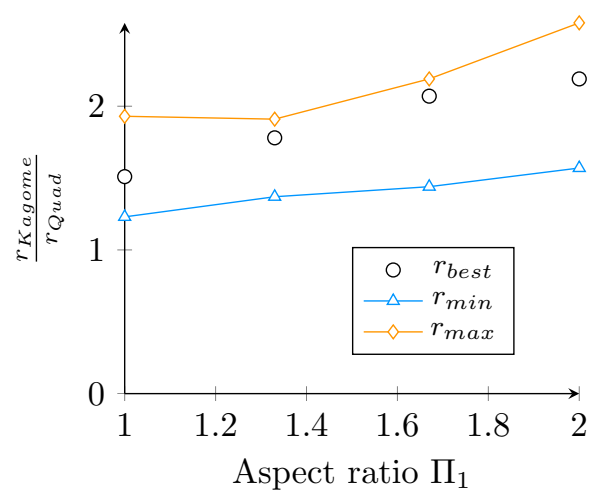

Figure 17: Comparison of the structural performance of domes under non-symmetrical load. in homogenised models by Lebée and Sab for flat thick quadrangular beams layouts [11].

$$
\left\{\begin{array}{l}
\mathcal{D}=\frac{E I}{l} \\
\mathcal{A}=\frac{E A}{l}
\end{array}\right.
$$

The buckling load of an isotropic spherical shell under uniform pressure $p_{c r}$ is given by following the formula, found for example in [35] or [34]:

$$
p_{c r}=\frac{2 \sqrt{\mathcal{A D}}}{R^{2}}
$$

By combining equations (13) and (14), the critical buckling load of an equivalent isotropic shell depends thus linearly on the number of cells. It is well-known that homogenised models describe accurately the actual model when the number of cells is large enough. Having these considerations in mind, we should expect the ratio $\Pi_{5}$ to be constant for sufficiently small values of $\Pi_{2}$, because the mass $m$ varies linearly with $1 / l$ and so does the homogenized buckling load. Figure 20 shows the variations of the structural efficiency with respect to $\Pi_{3}$. It appears that $\Pi_{5}$ tends to a constant when $\frac{1}{\Pi_{3}}$ increases. The convergence is reached for $\frac{1}{\Pi_{3}} \simeq 25$. This value is linked with the chosen cross-section, here $200 \mathrm{~mm}$ pipes. The slenderness of the members at $\frac{1}{\Pi_{3}} \simeq 25$ is of 5 , which is higher than what is found in built projects.

The convergence of the structural efficiency to a constant indicates that kagome grids tend to behave like isotropic shells. On the contrary, the efficiency of the quadrilateral grid does not converge to a constant when the grid is refined. To better understand this, consider that the buckling capacity of orthotropic shells depends on the in-plane shear stiffness. An example of analytical formula is given for shells of revolution in [34]. Still referring to the results of Lebée and Sab [11], we give an estimate of the in-plane shear stiffness $G_{x y}$ for quadran- 


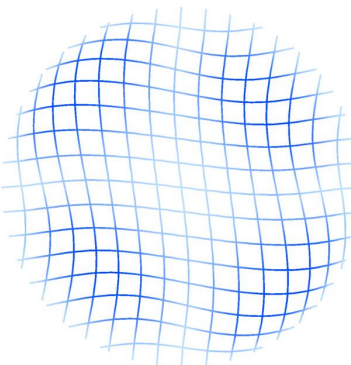

(a) First mode, $\Pi_{4}=889$
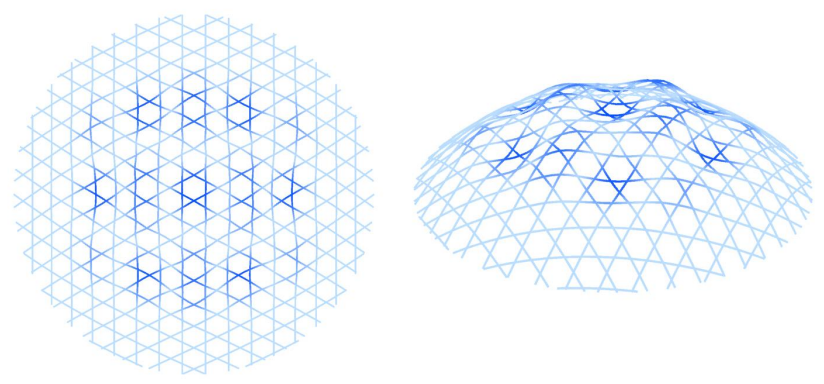

(b) First mode, $\Pi_{4}=6885$

Figure 18: Comparison of the first buckling modes for quadrilateral and kagome meshes.

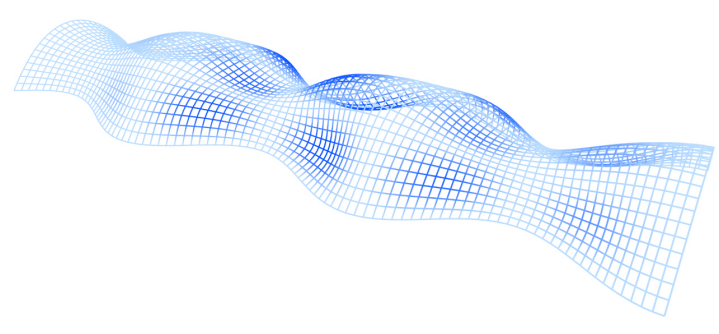

(a) First mode, $\Pi_{4}=6145$

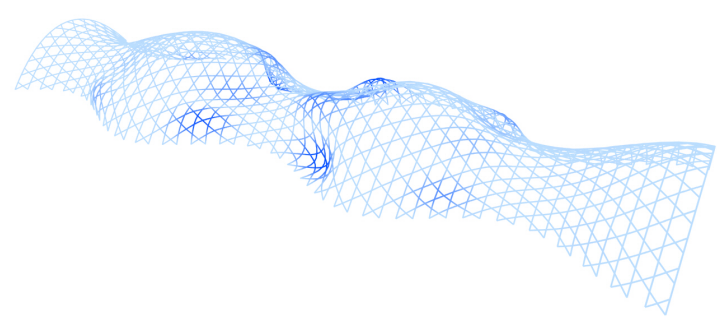

(b) First mode, $\Pi_{4}=9361$

Figure 19: Comparison of buckling modes on the most performant barrel vaults in our study.

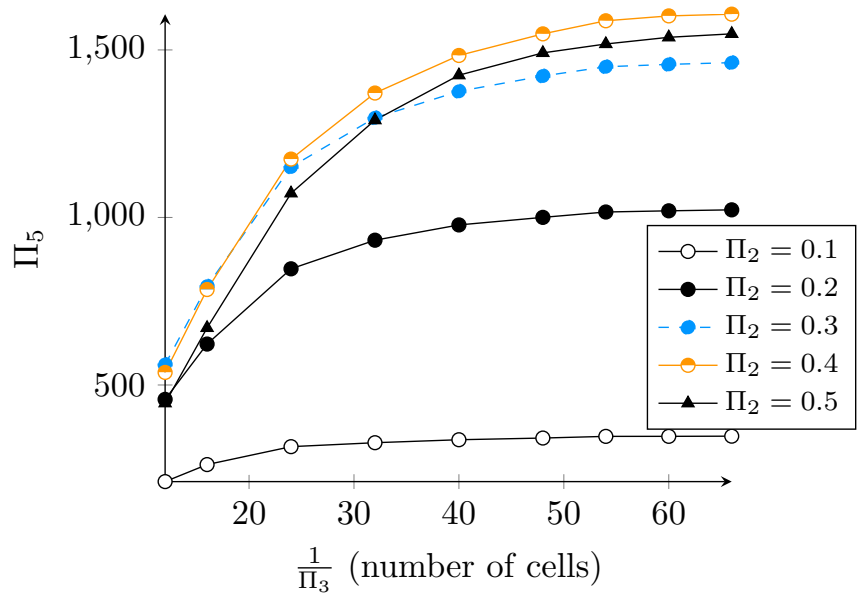

Figure 20: Structural efficiency for different refinements of a kagome grid $\left(\Pi_{1}=1.33\right)$. gular grids (we make the assumption that the beams are Euler-Bernoulli beams):

$$
G_{x y}=\frac{E I}{l^{3}}
$$

This term clearly increases faster than the bending stiffness when the unit-cell becomes smaller. This nonlinearity explains why the graph of Figure 21 increases with the number of cells $\frac{1}{\Pi_{2}}$ without reaching a plateau. The convergence study is thus a good indicator of the fact that kagome gridshells are isotropic, whereas quadrilateral grids are orthotropic.

\subsection{Design guidelines for kagome gridshells}

Structural engineers can improve the efficiency of their designs by using different strategies. We discuss here some of these. In our study, the shape is an important factor of performance: changing a rise-over-span ratio from $10 \%$ to $20 \%$ doubles the structural efficiency. The optimal rise-over-span ratio is around 30\%. For larger values, the gridshells become subject to localised buckling, and overall curvature of the shape does not provide any help. The change of geometry does not bring significant changes in the cost of fabrication of the elements, as our method guarantees meshing with flat panels.

Increasing the structural density also increases the structural performance of quadrilateral and kagome grids. For kagome grids, this strategy has a limit, as the structural efficiency tends to a constant when the number of 


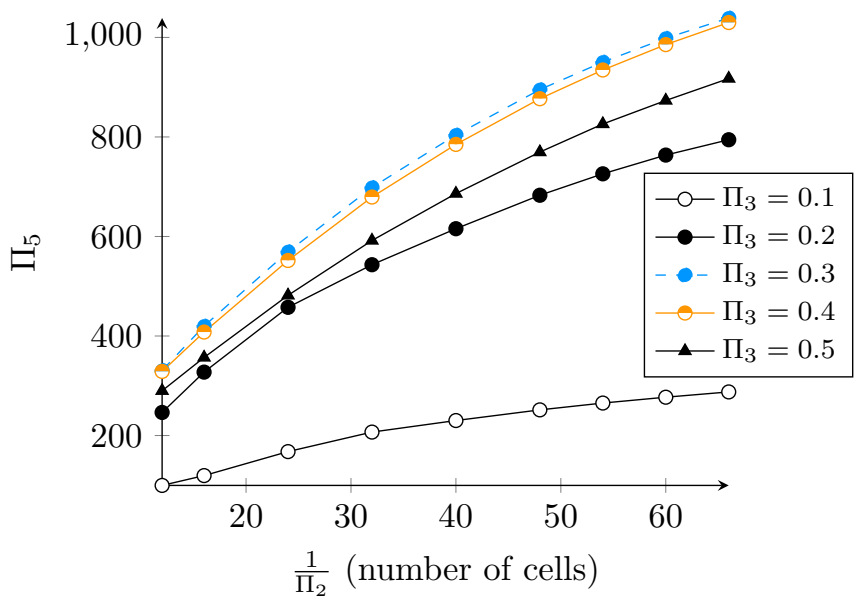

Figure 21: Structural efficiency for different refinements of a quadrilateral grid $\left(\Pi_{1}=1.33\right)$.

cells tends to infinity. Even for very high structural density, this phenomenon does not occur for the quadrilateral grids studied in this paper. This strategy has however a practical limitation, as the number of connections increases when increasing the density of the grid. Connections are very expensive and often govern the cost of the structure in gridshells. Denser grids are also costly, and the benefit in structural performance might be tempered by an increased construction cost.

We note here that the conversion rule chosen implies that, for a same value of $\Pi_{2}$, kagome grids have less vertices (and thus connections) than quadrilateral grids. Writing $N_{\text {Kagome }}$ and $N_{\text {Quad }}$ the number of nodes, we have following simple relation proven in Appendix A:

$$
\frac{N_{\text {Kagome }}}{N_{\text {Quad }}}=\frac{\sqrt{3}}{2} \simeq 86 \%
$$

Therefore, the kagome grids of the present study are structurally more efficient than quadrilateral grids for gridshells designed with a linear buckling criterion, and their cost of connections is also significantly lower.

\section{Conclusion}

This article has introduced a method to cover kagome meshes with planar facets. This simplifies the fabrication of the cladding, a major concern in free-form architectural design. The bearing capacity of kagome gridshells was then studied and compared to the one of quadrilateral gridshells. The results seem promising, as the kagome gridshells has a significantly higher performance in our case studies, both for symmetrical and non-symmetrical load cases. The better performance of kagome grid pattern seems to come from its higher in-plane shear stiffness. The gain in structural efficiency compared to quadrilateral gridshells is higher when biaxial stresses are at stake in the structure.
The gridshells were not compared to other common solutions, like cable-braced or triangular gridshells. While these solutions are probably structurally more efficient than kagome grid patterns (a comparison between kagome and triangular pattern for a GFRP gridshell has been done in [36], and shown that triangular pattern can be two or three times stiffer than kagome grid pattern), they are also more complex to build, due to high node valence or tuning of cable tension. Steel contractors might prefer to build moment connections rather than installing cables [4].

The study proposed in this paper considered mainly linear buckling analysis, a tool suited for the exploration of the design space in preliminary phases of design. The influence of imperfections was however discussed and showed that, like triangular grids, kagome grids are sensitive to geometrical imperfections. Some parameters were not considered in our studies: introducing realistic node stiffness and material non-linearities in the parametric study could greatly improve the estimation of real collapse loads and would provide in further work reliable values for detailed design.

\section{Acknowledgment}

We want to thank the anonymous reviewers who contributed to the improvement of this paper with constructive remarks. The authors also appreciate preliminary calculations by Fernando Vianna Brasil Medeiros and Leandro Dos Reis Lope, both graduate students at l'École National des Ponts et Chaussées. Finally, the authors thank Charis Gantes (NTUA) for fruitful discussions that led to the introduction of the simplified SLS criterion used in this paper.

This work was made during Mr. Mesnil doctorate within the framework of an industrial agreement for training through research (CIFRE number 2013/1266) jointly financed by the company Bouygues Construction SA, and the National Association for Research and Technology (ANRT) of France.

\section{Appendix A. Basic properties of kagome grid pat- tern}

The kagome grids generated in our study tend to have a uniform member length. We propose simple calculations to estimate the number of connections or member length per unit area for a planar kagome grid made out of regular hexagons and triangles.

\section{Appendix A.1. Description of the pattern}

The regular kagome pattern is made out of regular hexagons and triangles. The pattern is periodic, and can thus be described by the study of a unit-cell shown in Figure A.22. In this image, all the edges have the same length $l$, the dimensions of the unit cell are easily found based on properties of equilateral triangles. 


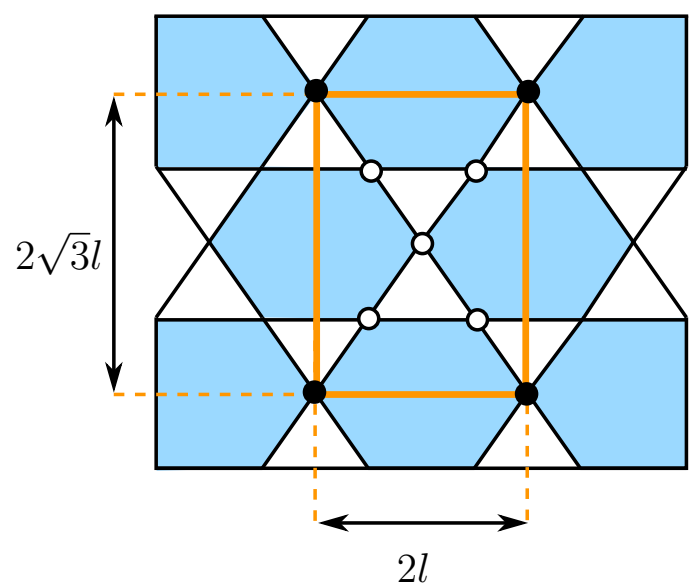

Figure A.22: A basic cell of a kagome grid.

The pattern is compared to a square pattern, where the unit cell is obviously a square with edge length $l$.

\section{Appendix A.2. Structural density}

We compute now the edge length per unit area. In the unit cell, we count 10 edges and 4 half edges. The edge length per unit area $L_{A}$ is thus:

$$
L_{A}=\frac{\left(10+4 \cdot \frac{1}{2}\right) \cdot l}{2 \sqrt{3} l \cdot 2 l}=\frac{\sqrt{3}}{l}
$$

We can compare this value with the edge length per unit area for the square pattern, where $L_{A}=\frac{2}{l}$. For a same edge length, the ratio of member lengths is thus equals to $\frac{\sqrt{3}}{2}$. This gives the estimation for the mass ratio of equation (8).

\section{Appendix A.3. Number of connections}

The number of nodes per unit area is an important question, as the cost of connections highly impacts the cost of gridshells. For the unit cell depicted in Figure A.22, there are 5 nodes that belong only to the cell (in white), whereas 4 nodes belong to 4 adjacent cells (in black). The number of connections per unit area is thus:

$$
N_{\text {nodes }}=\frac{5+4 \cdot \frac{1}{4}}{2 \sqrt{3} l \cdot 2 l}=\frac{\sqrt{3}}{2 l^{2}}
$$

For a square grid, the number of nodes per unit area is simply $\frac{1}{l^{2}}$. The ratio of these two values is thus equals to $\frac{\sqrt{3}}{2}$, which gives an estimate for the ratio used in equation $(16)$.

\section{Appendix B. Homogenisation approach and equivalent buckling loads}

In this Section, we adapt the formula of an anistropic spherical cap of radius $R$ under uniform pressure found by Crawford [34] to a quadrangular gridshell with equivalent properties derived from [11]. The problem treated by Crawford considers that the shell is isotropic with principal axis along parallel and meridians. The geometry is different from the domes studied in this paper, but it one of the only analytical formulæ available in the literature for orthotropic shells. We take count of the fact that our problem deals with circular hollow sections to simplify $I_{y}=I_{z}=I$.

\section{Appendix B.1. Equivalent shell stiffness of a quadrangular gridshell}

Let us construct the equivalent axial and bending stiffness tensors from the homogenisation of a quadrilateral grid. From [11], we have:

$$
\left\{\begin{array}{r}
A_{x x}=A_{y y}=\mathcal{A}=\frac{E S}{l} \\
G_{x y}=\left(\frac{l}{G \mathcal{S}}+\frac{l^{3}}{12 E I}\right)^{-1} \\
\nu_{x}=0 \\
\nu_{y}=0
\end{array}\right.
$$

and

$$
\left\{\begin{aligned}
D_{x x}=D_{y y}=\mathcal{D} & =\frac{E I}{l} \\
D_{x y} & =\frac{G J}{l}
\end{aligned}\right.
$$

The grid relies only on bending of elements for the inplane shear stiffness, and on beam torsion for the torsional stiffness of the equivalent shell. All terms depend linearly on $\frac{1}{l}$ (equivalently the number of cells) except the in-plane shear stiffness, which depends on $\frac{1}{l^{3}}$.

\section{Appendix B.2. Buckling of orthotropic spherical cap under uniform pressure}

We derive now the theoretical buckling load of an anistropic shell from the work of Crawford [34]. The equations simplify greatly when $D_{x x}=D_{y y}$ and $A_{x x}=A_{y y}$. Crawford introduces the quantities $D_{3}, G_{3}$ and $\Psi$ defined by:

$$
\left\{\begin{array}{r}
D_{3}=\nu_{x} \cdot D_{y}+D_{x y} \\
G_{3}=\frac{2 G_{x y}}{1-\nu_{x} \nu_{y}-\left(2 \nu_{y} \frac{G_{x y}}{A_{y y}}\right)} \\
\Psi=\frac{\mathcal{A}\left(1-\nu_{x}^{2}\right)}{\mathcal{D} R}
\end{array}\right.
$$

With these notations, the buckling load of the isotropic spherical shell is:

$$
p_{c r}=\frac{4 \mathcal{D} \sqrt{\Psi}}{R}
$$


Crawford computes then the ultimate buckling load of the anistropic shell $\overline{p_{c r}}$ given by:

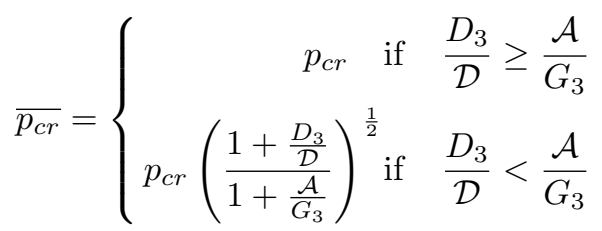

In the case of gridshells, the second inequality is verified, and using equations (B.1) and (B.2), we obtain:

$$
\overline{p_{c r}}=\frac{4 \sqrt{\mathcal{A D}}}{R^{2}} \sqrt{\frac{1+\frac{D_{x y}}{\mathcal{D}}}{1+\frac{\mathcal{A}}{2 G_{x y}}}}
$$

So that finally:

$$
\overline{p_{c r}}=\frac{4 E \sqrt{S I}}{l R^{2}} \sqrt{\frac{1+\frac{G J}{E I}}{1+E S\left(\frac{1}{2 G \mathcal{S}}+\frac{l^{2}}{24 E I}\right)}}
$$

The first term corresponds to the buckling capacity of an istropic shell, it is proportional to $\frac{1}{l}$. The second term (under the square root) varies nonlinearly with $\frac{1}{l}$ because of the term in $l^{2}$, which comes from the equivalent inplane shear stiffness. The limit of structural efficiency for a high number of cells is given by equation (B.8), as $l$ tends towards 0 . We write $\rho$ the volumic mass of steel (the mass per unit of a quad grid being $\frac{2 \rho}{l}$ ) and get:

$$
\Pi_{5}^{*}=\lim _{N \rightarrow \infty} \Pi_{5}=\frac{2 E \sqrt{S I}}{\rho R^{2}} \sqrt{\frac{1+\frac{G J}{E I}}{1+\frac{E S}{2 G \mathcal{S}}}}
$$

Figure B.23 shows the application of equation (B.7) with the cross-section used in our parametric study and $\Pi_{1}=1$, which is the closest configuration to a spherical cap. The limit of the structural efficiency $\Pi_{5}^{*}$ is also shown. It is noticed that the orthotropic shell converges slowly towards the limit, which explains why our convergence study does not show a plateau for the quadrilateral gridshell.

\section{References}

[1] Glymph, J., Shelden, D., Ceccato, C., Mussel, J., Schober, H.. A parametric strategy for free-form glass structures using quadrilateral planar facets. Automation in Construction 2004;13(2):187-202.

[2] Mesnil, R., Douthe, C., Baverel, O., Léger, B., Caron, J.F.. Isogonal moulding surfaces: A family of shapes for high node congruence in free-form structures. Automation in Construction 2015;59:38-47.

[3] Mesnil, R., Douthe, C., Baverel, O.. Marionette Meshes: from descriptive geometry to fabrication-aware design . In: Advances in Architectural Geometry. 2016,.

[4] Schober, H., Justiz, S.. Cabot circus, bristol ebene vierecknetze für freigeformte glasdächer. Stahlbau 2012;81(S1):28-42.

[5] Pottmann, H., Liu, Y., Wallner, J., Bobenko, A., Wang, W.. Geometry of multi-layer freeform structures for architecture. ACM Transactions on Graphics 2007;26(3):65. .

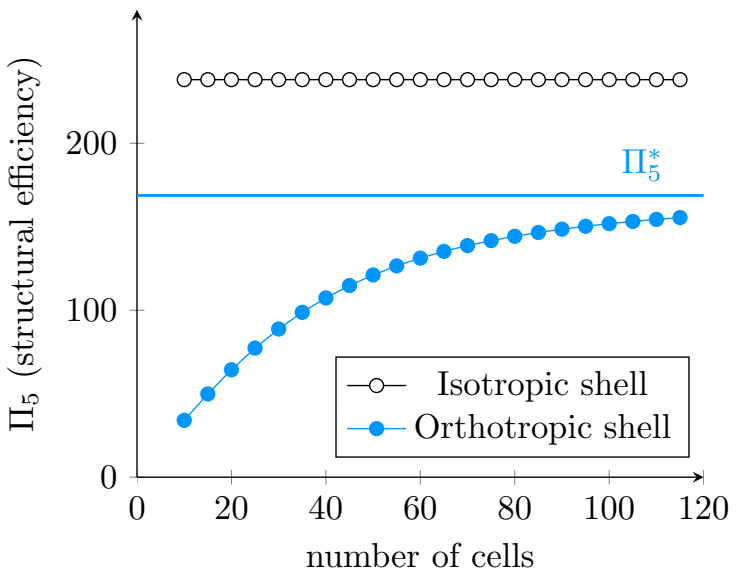

Figure B.23: Influence of grid refinements on the structural efficiency with a homogenised model

[6] Bulenda, T., Knippers, J.. Stability of grid shells. Computers \& Structures 2001;79(12):1161-1174. .

[7] Gioncu, V.. Buckling of reticulated shells: State of the art. International Journal of Space Structures 1995;10(1).

[8] Kato, S., Fujimoto, M., Ogawa, T.. Buckling load of steel single-layered reticulated domes of circular plan. Journal of the International Association for Shell and Spatial Structures $2005 ; 46(147): 41-63$.

[9] Galambos, T.V.. Guide to stability design criteria for metal structures. John Wiley \& Sons; 1998.

[10] Lebée, A., Sab, K.. A Bending-Gradient model for thick plates. Part I: Theory. International Journal of Solids and Structures 2011;48(20):2878-2888.

[11] Lebée, A., Sab, K.. Homogenization of a space frame as a thick plate: Application of the Bending-Gradient theory to a beam lattice. Computers \& Structures 2013;127:88-101. .

[12] Gioncu, V., Balut, N.. instability Behaviour of Single Layer Reticulated Shells. International Journal of Space Structures 1992;7(4):243-252.

[13] Buchert, K.P.. Split Rigidity Theory of Plates, Shells \& Stability. Columbia; 1985.

[14] Winslow, P., Pellegrino, S., Sharma, S.B.. Multi-objective optimization of free-form grid structures. Structural and Multidisciplinary Optimization 2010;40:257-269.

[15] Malek, S., Wierzbicki, T., Ochsendorf, J.. Buckling of spherical cap gridshells: A numerical and analytical study revisiting the concept of the equivalent continuum. Engineering Structures $2014 ; 75: 288-298$. .

[16] Lefevre, B., Douthe, C., Baverel, O.. Buckling of elastic gridshells. Journal of the International Association for Shell and Spatial Structures 2015;56(3):153-171.

[17] Kato, S., Mutoh, I., Shomura, M.. Collapse of semi-rigidly jointed reticulated domes with initial geometric imperfections. Journal of Constructional Steel Research 1998;48(2-3):145-168.

[18] Kato, S., Yamashita, T., Nakazawa, S., Kim, Y.b., Fujibayashi, A.. Analysis based evaluation for buckling loads of two-way elliptic paraboloidal single layer lattice domes. Journal of Constructional Steel Research 2007;63(9):1219-1227.

[19] Hwang, K.J., Knippers, J., Park, S.W.. Influence of Various Types Node Connectors on the Buckling Loads of Grid Shells. In: Proceedings of the International Association for Shell and Spatial Structures (IASS) Symposium 2009 Evolution and Trends in Design, Analysis and Construction of Shell and Spatial Structures. October; 2009, p. 1841-1852. .

[20] Bruno, L., Sassone, M., Venuti, F.. Effects of the Equivalent Geometric Nodal Imperfections on the stability of single layer grid shells. Engineering Structures 2016;112:184-199.

[21] Mesnil, R., Douthe, C., Ochsendorf, J.. Stability of Pseudo- 
Funicular Elastic Grid Shells. International Journal of Space Structures 2015;30(1):27-36.

[22] Tayeb, F., Caron, J.F., Baverel, O., Du Peloux, L.. Stability and robustness of a $300 \mathrm{~m} 2$ composite gridshell structure. Construction and Building Materials 2013;49:926-938.

[23] Koiter, W.T.. The stability of elastic equilibrium. Tech. Rep.; Air Force Flight Dynamics Laboratory; 1977. .

[24] Knippers, J., Helbig, T.. Recent Developments in the Design of Glazed Grid Shells. International Journal of Space Structures 2009;24(5):111-126.

[25] Schlaich, M., Irisarri, L., Goñi, J., Burkhardt, U.. Palacio de comunicaciones-a single layer glass grid shell over the courtyard of the future town hall of madrid. In: Symposium of the International Association for Shell and Spatial Structures (50th. 2009. Valencia). Evolution and Trends in Design, Analysis and Construction of Shell and Spatial Structures: Proceedings. Editorial Universitat Politècnica de València; 2010,.

[26] Sischka, J.. Engineering the construction of the great court roof for the British Museum; chap. 21. Thomas Telford; 2000, p. 199-207. .

[27] Yamada, S., Takeuchi, A., Tada, Y., Tsutsumi, K.. Imperfection-sensitive overall buckling of single-layer lattice domes. Journal of Engineering Mechanics 2001;2(April):382386.

[28] Harris, R., Romer, J., Kelly, O., Johnson, S.. Design and construction of the downland gridshell. Building Research \& Information 2003;31(6):427-454.

[29] Preisinger, C., Heimrath, M.. Karamba - A Toolkit for Parametric Structural Design. Structural Engineering International 2014;24(2):217-221. .

[30] Preisinger, C.. Linking Structure and Parametric Geometry. Architectural Design 2013;83(2):110-113. 10.1002/ad.1564.

[31] Bobenko, A., Suris, Y.B.. Discrete Differential Geometry: Integrable Structure. American Mathematical Society; 2008. .

[32] Liu, Y., Wang, W., Pottmann, H., Wallner, J., Yong-Liang, Y.. Geometric Modeling with Conical Meshes and Developable Surfaces. ACM Transactions on Graphics 2006;25(3):681-689.

[33] Mesnil, R., Douthe, C., Baverel, O., Léger, B.. Generalised cyclidic nets: an alternative to NURBS for the modeling of complex shapes in architecture. Computer-Aided Design 2016;

[34] Crawford, R.F., Schwartz, D.B.. General instability and optimum design of grid- stiffened spherical domes. AIAA Journal 1965;3(3):511- -515 . .

[35] Timoshenko, S., Gere, J.. Theory of elastic stability. New York: McGraw-Hill; 1961.

[36] Douthe, C., Caron, J.F., Baverel, O.. Gridshell structures in glass fibre reinforced polymers. Construction and building materials 2010;24(9):1580-1589. 